\title{
Research on the construction of SAP business intelligence virtual experiment teaching project
}

\section{Wang Lufang, Zhang Zhijun, Xu Huihong}

Experimental Teaching Center, Shanxi University of Finance \& Economics, Taiyuan, 030006, China

Keywords: SAP Business intelligence; Experimental teaching; Virtual projects; Virtual network platform

\begin{abstract}
This article is based on the construction of the SAP business intelligence virtual experiment teaching project for Shanxi University of Finance \& Economics, according to the construction background of this virtual teaching project, It deeply analyzes the current development of business intelligence technology, discussed how to achieve the target of training compound talents and the necessity of the virtual project construction. Then it gives the basic thought and realization of the virtual teaching project, finally introduces the framework design and implementation of the project in detail, It points out the development direction for further improving the teaching quality of the virtual project and improving the social service ability of the project in the future.
\end{abstract}

\section{基于SAP商务智能虚拟实验教学项目建设研究}

\author{
王路芳, 张志军, 续慧泓 \\ 山西财经大学, 太原 山西 中国 030006
}

关键词: SAP; 商务智能; 实验教学; 虚拟项目; 虚拟网络平台

摘 要: 本文以山西财经大学 SAP 商务智能虚拟实验教学项目建设为研究对象, 从该虚拟实 验教学项目的建设背景出发, 深入分析了以商务智能发展前沿和满足经管类复合型人才的培 养目标, 建设该虚拟项目的必要性。并进一步分析了虚拟实验教学项目在建设过程中的基本 思路及实现路径, 提出了建设项目的设计框架及具体实现, 同时对提升虚拟项目教学质量, 提高项目的社会服务能力提出建议。

\section{1. 引 言}

以“大智移云物”为代表的新一代信息技术的兴起, 对传统的商业模式正在产生颠覆性影响, 传统企业管理活动中的决策行为、决策方法和决策模式也随之发生变化。以经济学、管理学 为主干学科的财经类院校在培养具有较高水平的企业管理人才过程中，也同样面临着观念、 方法、技术和教学组织方面的创新。本文以山西财经大学基于SAP的商务智能虚拟实验教学 项目的建设为案例, 研究在新IT环境下, 借助于信息技术构建商务决策虚拟环境, 提升经管 类专业学生创新思维能力、科学决策能力、综合应用能力的途径和方法。

\section{2. 商务智能虚拟实验教学项目的建设背景}

在传统的管理信息系统中，商务智能（BI，Business Intelligence）是一套完整的解决方案， 用来将企业中现有的数据进行有效的整合, 快速准确的提供报表并提出决策依据, 帮助企业 做出明智的业务经营决策。商业智能的概念最早在1996年提出, 当时将商业智能定义为一类 由数据仓库（或数据集市）、查询报表、数据分析、数据挖掘、数据备份和恢复等部分组成 
的、以帮助企业决策为目的技术及其应用。商务智能的出现，使企业的决策更具科学性和可 验证性, 从而为提高管理效率奠定基础。管理和核心是决策, 掌握科学的决策方法和技术是 高素质企业管理人才必备的能力。因此，商务智能的相关实训一直是经管类专业学生必修的 课程内容之一, 但随着商务智能自身的发展演变和学校专业培养目标要求的不断提高, 如何 构建面向经管类专业的商务智能实训体系成为必须要解决的课题。

\section{1 商务智能发展对构建商务智能虚拟实验教学项目的影响}

\subsection{1 商务智能功能的扩展}

传统商务智能以数据分析、数据挖掘、数据可视化为基本需求, 实现从数据到信息、从信 息到知识、从知识到决策的转化。它是在原有业务系统的开发技术之上架构起来的，这些技 术很大程度上是专门服务于原有业务系统的运作模式, 往往存在移植性、集成性和可扩展性 差等缺点, 在商业需求频繁变化的条件下很难提供充分可靠的决策支持。现代商务智能更强 调数据展现向动态决策、实时决策的扩展，来弥补传统实现方式的缺陷。

\subsection{2 大数据方法的引入}

传统大数据研究对象主要是针对结构化、高度综合的数据集，随着信息技术的不断进步， 信息的存在结构越来越呈现多样性, 信息的主要来源从传统的企业内部逐渐延伸到整个互联 网, 信息以文本、音频、视频等等非结构化数据形式存在。因此对信息的研究从传统结构化 的数据逐渐向非结构化数据进行转变, 同时信息的容量也呈现爆炸式的增长, 每天会产生海 量信息，同时也存在巨量的垃圾信息，如何从海量信息中提取有助于企业决策的信息是当前 数据研究的主要方向。同时在研究方法上也从传统的因果分析方法向相关性分析方法转变, 更有利于挖掘潜在的商业价值。

\subsection{3 由独立系统向嵌入系统的过渡}

传统的商务智能研究方法, 是从原有数据库中抽取数据, 建立以分析主题为目标的、独立 的数据仓库, 然后再在数据仓库基础之上以主题板块划分进行可视化分析展示所形成的商务 智能系统是独立于原系统存在的，由于所抽取的数据是阶段性、非及时性的数据，所以在某 些强调及时性的特殊行业, 辅助决策上存在一定的滞后性, 难以完全满足客户的决策需求。 因此, 产生了另外一种嵌入式系统, 嵌入式系统直接运行在原生系统中, 其决策的数据来源 于实时发生的交易数据。但嵌入式系统在一定程度上会让整个管理系统过于庞大，是其不利 的一个方面。

\subsection{4 决策和控制过程的融合}

商务智能最大的优势就是能够较为及时的给客户提供辅助决策，但该决策仍然存在一定的 人为因素，因此可能存在根据决策来进行控制整个过程的滞后性，因此现代商务智能力求实 现决策和控制过程的无缝融合，实现实时动态的商业决策、控制、反馈及优化调整。

\section{2 专业培养目标对构建商务智能虚拟实验教学项目的影响}

\subsection{1 多学科交叉融合}

商务智能系统涉及管理学、经济学、计算机科学等多学科的综合应用, 因此在构建商务智 能虚拟实验教学项目上, 力求使不同专业背景的学生交叉成组进行讨论和实现, 有利于培养 适合社会发展需求的复合型、综合性人才，同时有利于提升虚拟实验项目的过程体验，使虚 拟实验项目有更好的决策度。

\subsection{2 综合思维能力和创新能力需求的提升}

商务智能虚拟实验教学项目的实现不但需要多学科知识的融合, 更需要学生综合思维能力, 在每个商业主题的实现过程中, 需要学生具备综合思维的能力, 因此在项目的实现过程中, 
能够很好的训练学生的综合性思维。同时虚拟实验项目实现的没有固定模式做限制, 学生可 最大限度的发挥各自的想象力和创造力, 自主创新完成相关任务。

\subsection{3 抽象模拟向具象实践的转变}

商务智能实验项目的构建方式分三个阶段，第一阶段，以演示型、验证型实验为主，学生 按照标准操作流程完成实验过程; 第二阶段, 以仿真模拟为主要手段, 以综合型实验为主, 通过高度抽象和总结的决策模型或决策场景, 让学生掌握决策方法, 体验决策流程; 第三阶 段, 以具体应用场景为背景, 以设计型、开放型实验为主, 让学生针对具体实践问题借助于 真实的软件平台，训练综合解决实际问题的能力，提升实践水平。

\section{3 商务智能虚拟实验教学项目的建设的必要性分析}

由以上分析可知, 商务智能虚拟实验教学项目建设要解决的关键问题是:

\subsection{1 复杂企业管理场景的模拟}

当前, 各院校在校学生数量众多, 大部分高校在有限的校外实习基地很难满足实际的实践 需求。这在很大程度上影响了学生的未来求职机会。为让学生在步入工作岗位前, 尽可能熟 悉未来的业务场景，提升未来快速适应工作的能力，提高毕业求职的高度和准确性。

商务智能虚拟实验教学项目在设计上力求最大程度接近企业的实际业务场景, 使企业实际 的核心场景在虚拟项目中得到最大程度的体现。学生进而能够在贴近现实的虚拟化场景中, 利用自身的专业素养, 发挥自己的想象力和创造力, 协力开发出能够满足基本决策需求的系 统。

\subsection{2 企业复杂变量决策过程的模拟}

商务智能决策是一个复杂的、综合性的过程, 其决策的结果往往依赖于多个重要指标变量 值。所以在具体的项目实现过程中, 需要对相关指标的影响因子做充分的定性定量分析, 根 据不同的场景，给予各指标合理的权重，从而满足在不同场景下产生适当的决策值。

\subsection{3 学生决策思维和决策过程的模拟}

学生完成虚拟教学项目往往需要一个反复的过程。在此过程中, 各岗位角色的学生依据自 身的专业背景, 对所要分析的主题进行分解, 列出各主题下相关的关键指标, 然后设定部分 核心指标的阈值。在经营过程中，部分阈值一旦有警示信息，需要深入考虑报警提示的影响 因素, 并及时作出相关的经营调整。在此过程中, 学生须熟悉各主题下相关指标的关联度, 同时使自身的决策思维得到提升。

\section{3. 商务智能虚拟实验教学项目的建设的基本思路}

\section{1 开放设计}

\section{第一，选题开放}

不拘泥于具体决策问题或既定的决策案例, 学生自主选题, 以企业实践问题为导向, 针对 问题设计主题，并找出相关主题下的关键指标，确定各指标存在的数据表之间的关系，创建 相关主题下的数据集市。

\section{第二，方法开放}

不拘泥于固定的决策方法和过程，由学生自主选择不同的方法对各自的问题展开分析。最 终学生在相同或相似主题下, 运用不同的方法展开讨论分析, 来发现针对相似主题的最优决 策方法和过程。

第三，平台开放

借助于开放的虚拟化实验平台完成线上、线下应用的融合，使课堂教学、课下实践在线上 和线下得以充分展开, 并在此基础上对学生的项目进度进行跟踪指导, 及时发现虚拟实验项目 
的不足并加以改进, 同时对学生的项目进度加以监督和推进, 确实让学生在项目实践中提升 专业能力、创新能力和综合素质, 从而全面形成新的实验教学模式, 有利于高校创新人才的培 养。

\section{2 实践导向}

商务智能虚拟实验项目的设计, 不应该局限于某种固定的模式和流程, 让学生照搬来进行 设计。而应该从企业的实际问题出发, 通过前期问题的调研、讨论, 然后大家根据不同的理 解来确认自己的决策主题、决策方法以及决策流程。所以在课堂教学的过程中不是教学过程 的模拟, 而是真实的企业商务智能构建和应用场景。以解决实际问题为目标, 将商务智能的 实践项目融入实训中来。

\section{3 虚拟平台}

网络虚拟化平台采用虚实结合、软硬结合、远近结合的构建方式，能充分满足不同专业、 不同层次实训目标的需求, 参加实训的学生可以在校内外任何时间、任何电脑上对所需实现 的虚拟项目资源进行访问, 可以下载实验课件、汶览实验大纲、学习慕课课程、熟悉实验流 程、通过网络社区与同学或者指导教师进行交流。同时指导教师可发布新的实验案例, 上传 实验教材或者慕课课程, 及时更新教学资源。也可通过网络对学生的虚拟项目进行跟踪和监 督, 在线回答解决问题, 指导实验操作, 对学生的实验结果给出评价, 在网上批阅实验报告, 并能够将批阅的报告发给相应学生。

\section{4. 商务智能虚拟实验教学项目的建设的实现路径}

\subsection{SAP 大数据及商务智能平台的引入}

SAP于1972年在德国创立, 是全球最大的企业管理和协同化商务解决方案供应商, 提供优 质的应用程序和服务, 帮助超过25个行业内各种规模的企业实现卓越运营。SAP将企业软件 定义为企业资源计划、商务智能和相关应用程序的集合。其核心业务是销售其研发的商业软 件解决方案及其服务的用户许可证。SAP解决方案包括标准商业软件及技术以及行业特定应 用, 主要目标是帮助企业建立或改进其业务流程, 使之更为高效灵活, 并不断为企业产生新 的价值。

山西财经大学实验教学中心作为SAP大学联盟中国区九个加速器之一, 积极引入SAP BO (SAP BusinessObjects) 商务智能开发套件, 并与SAP展开多层次深入合作。在实验课程设计 上, 涉及商务智能项目整个开发流程所设计的数据仓库、数据集市、可视化设计等三类工具 的初级应用。

\subsection{BI 企业应用案例库的构建}

企业应用案例库构建来源于三种方式:

第一、基于SAP提供的最佳企业实践。SAP在软件推广过程中, 经常总结软件实施中的经 典案例, 这些经典案例涵盖了众多典型应用场景, 对这些案例的学习, 将极大提高学生对商 务智能内涵的理解及其实践应用的能力。

第二、教师科研项目的植入。结合教师团队对不同行业的商务智能应用和研究, 借助于SAP 商务智能开发套件, 教师在完成自己科研的同时, 将研究成果共享到企业应用案例库中, 供 校内师生共同研究学习。

第三, 通过学生开放项目的遴选, 学生在完成虚拟实验案例项目的同时, 会产生许多优秀 的案例, 将这些案例进行统一修订, 分享到应用案例库中供大家学习, 另外在九月份和SAP 联合举办的全国SAP商务智能大赛,遴选许多优秀商务智能项目案例来充实企业应用案例库。 


\section{3 线下课程与线上虚拟的嵌入}

按照虚拟实验教学项目的建设要求, 实现线下课程和线上虚拟资源的嵌入和融合。线下课 程植入企业资源计划综合实训课程中, 主要任务是引导学生掌握基本的方法和工具, 并启发 学生按照项目方式自行选题、组建团队, 介绍完整商务智能项目开发过程、SAP BI开发工具 的体系架构。

线上虚拟资源包括线上辅助学习、视频课程、互动社区、在线辅导、开发工具平台等资源, 学生可以通过网络平台登录并访问相关资源, 获得在线支持和辅导。

\section{5. 商务智能虚拟实验教学项目设计与实现}

\section{1 整体框架设计（如图 1 所示）}

商务智能虚拟实验教学项目框架涵盖三大的模块, 辅助支持平台、应用支持平台及基础资 源平台。

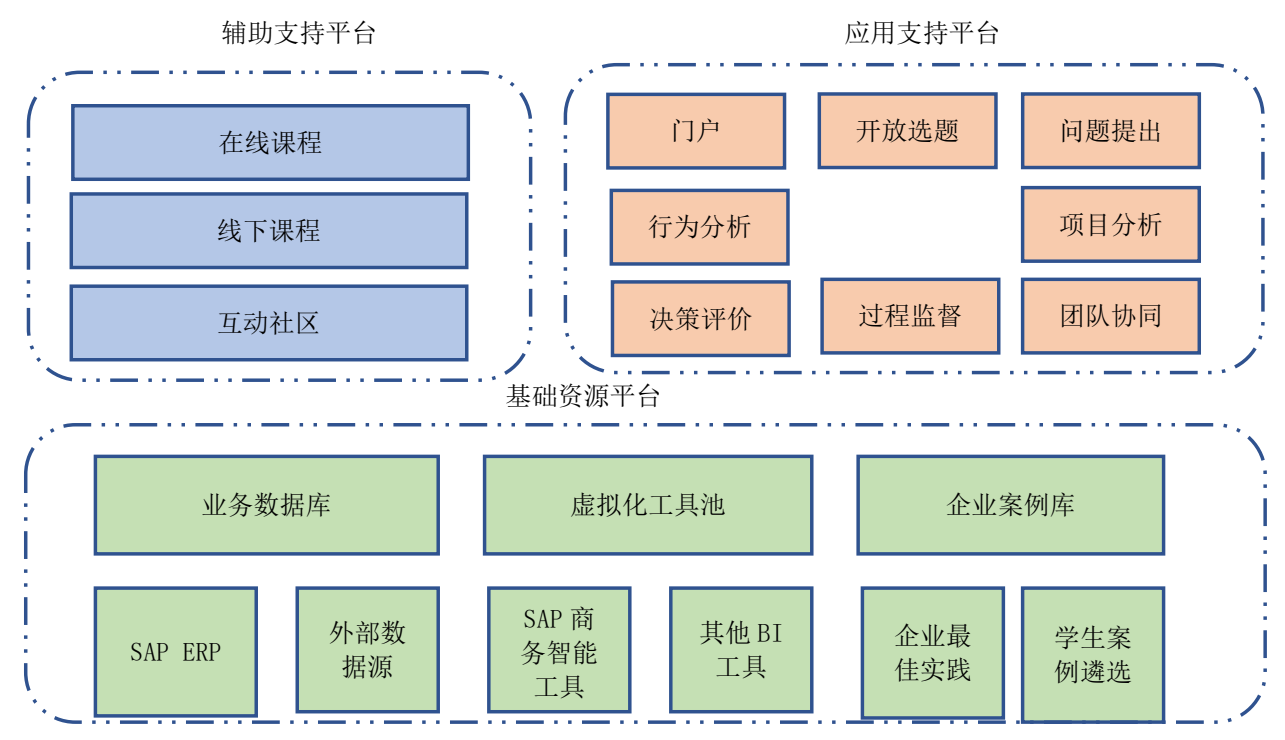

图1 商务智能虚拟实验教学项目整体框架

辅助支持平台: 辅助完成基本的教学任务, 在该平台学生能够对线上和线下开设课程完成 学习, 并在互动社区对课程相关主题设计实现进行讨论。

应用支持平台：作为商务智能虚拟项目的延伸, 要求学生自主开放选题, 然后自由组建团 队, 来完成选题设计。在此过程中, 需要对各开放主题进行分析指导、过程监督, 同时各团 队需展开紧密的团队协作，来共同完成选题。

基础资源平台：给学生提供大量的商务智能的相关资源，比如精简化的企业业务数据库、 多种商务智能开发工具池、优秀案例库集等等众多学习资源。通过对这些资源的广泛学习来 提高自主选题的完成质量。

\section{2 教学组织}

教师组织: 实验教学中心下设的ERP教研室, 由来自于工商管理学院、管理工程学院、会 计学院、信息学院等17位兼职教师和6位专职教师组成, 学生团队的自主选题需要得到多学科 知识的支持和指导, 多学科团队的构成对虚拟实验教学项目的顺利开展提供了有力的保障。

学生组织: 参加虚拟教学项目的学生来自于经管类学院, 在虚拟项目完成团队上实行跨学 科学生团队组建，同时实行项目式组织管理，便于虚拟实验项目的顺利推进。

项目设计: 线下4课时的设计, 课时植入ERP综合实训课程; 线上开放, 以ERP案例、企业 案例库为参照自主选题, 个性化完成。 
教学管理: 引入项目化管理方式, 加强线上持续跟踪, 通过案例大赛和学生参与教师科研 给予激励。

\section{3 课程设计}

第一，问题提出

团队成员对选题进行所属行业分析讨论, 找出该行业的关注点, 设计分析主题及主要分析 内容指标;

\section{第二，方法选择}

针对不同的分析主题，讨论研究主题相关数据构成，初步确定分析方法;

第三，建立语义层

使用SAP IDT(信息设计器)进行语义设计, 建立数据基础, 设计表间关系, 按照业务需求重 新划分类，最后定义维度对象、度量对象和特征对象，并检验数据的完整性约束;

第四，原型展示

团队成员按主题进行分工，利用SAP Lumira做初步的可视化实现;

第五, 互动讨论

同类主题的团队间进行讨论，对实现方案进行反复讨论，确定各主题数据最优展示形式。

\section{4 虚拟技术的应用}

为保证学生线上和线下学习对服务端的访问流畅性, 我们将整个商务智能虚拟实验项目的 学习资源发布到校园实验云平台, 并在云平台提供SAP BO商务智能套件客户端、数据仓库工 具SAP Data Services客户端、Tableau、帆软FineBI等主流商务智能的开发平台，供学生下载体 验学习。

\section{6. 商务智能虚拟实验教学项目效果评价}

第一，目前已进入试运行阶段，通过商务智能虚拟实验教学项目的引入，打通了原有课程 的界限和专业限制, 不同专业项目团队成员的交叉融合有助于学生专业综合能力的培养。

第二, 学生通过该实验项目, 培养数据分析的思维, 深入了解商务智能的内涵及价值, 提 升应用新技术和新方法的能力，提高了实践应用能力，为未来快速适应工作环境奠定基础。

第三，学生深度投入，教学效果良好。校内经管类学生实验相对有限，专业内所开设实验 基本都是基于专业课程的延伸学习, SAP 商务智能虚拟教学项目作为一个涉及多学科的综合 性实验, 在先期的试运行阶段受到学生们的广泛好评。

\section{7. 商务智能虚拟实验教学项目应用展望}

该虚拟实验教学项目仍然在初期建设和试运行阶段，随着实验项目逐渐成熟，后期还有大 量的工作需要推进:

第一，继续加深同SAP的项目合作，实验教学中心正研究部署SAP HANA， SAP HANA支 持企业预置型部署和云部署模式, 提供高性能的数据查询功能, 用户可以直接对大量实时业 务数据进行查询和分析, 而不需要对业务数据进行建模、聚合等。这个工具的引入会大大提 升学生对大数据的数据分析和实践应用能力。

第二，积极开展对外服务，建立项目合作共建院校，将项目资源分享给共建院校，共同充 实虚拟实验项目的教学资源。同时积极开展校企合作, 真正让学生在走出校门前, 对企业应 用有更直观的体验学习, 在力所能及的范围内将所学服务于企业和社会。

\section{[参 考 文 献]}

[1] 邬贺铨. 新一代信息技术的发展机遇与挑战[J]. 中国发展观察, 2016(4):11-13. 
[2] 刘玉波. 基于ESL的大数据商业智能系统的研究与实现[D]. 广东工业大学, 2015.

[3] 张川, 王圭金金. 商业智能中的系统集成探究[J]. 城市建设理论研究:电子版, 2013(36).

[4] Gebeyehu Belay Gebremeskel. 面向商业智能的数据挖掘算法和多智能体系统的体系结构 以及优化[D]. 重庆大学, 2013.

[5] 张全武. 基于Web Service技术的商业智能体系结构研究[D]. 大庆石油学院, 2007.

[6] 吴玉婷. 连锁零售业电子商务大数据发展研究[D]. 云南大学, 2015.

[7] 董雁适, 程翼宇, 潘云鹤. 基于逐步条件相关性分析的因果关系发现方法[J]. 模式识别与 人工智能, 2002, 15(4):000478-483.

[8] 王军. 数据仓库技术在商业智能系统中的应用[D]. 华东师范大学, 2007.

[9] 郑炎. 基于数据仓库的商业智能系统研究与实践[D]. 南京工业大学, 2005.

[10] 张丽. 企业商务智能决策支持系统的构建研究[D]. 安徽农业大学, 2008.

[11] 薛云. 应用商务智能系统推进企业管理信息化[J]. 中国商论, 2012(2):120-120.

[12] C. 维塞尔斯, John, Wiley,等. 商务智能用于决策的数据挖掘与最优化 $[\mathrm{J}]$. 国外科技新书评 介, 2009(9):2-3.

[13] 朱辉, 刘北水, 李晖,等. 基于虚拟化技术的信息安全实验平台开发与应用 $[\mathrm{J}]$. 武汉大学 学报(理学版), 2012(s2):249-252.

[14] 罗乔欣. 世纪转型之SAP SAP重新起飞[J]. 电子商务, 2000(7):46-49.

[15] 张得牛. SAP与电子商务系统集成的研究与实现[D]. 上海交通大学, 2011.

[16] 许航. 基于Sap Hana内存计算的大规模数据分析系统的设计与实现[D]. 吉林大学, 2016. 